\title{
Making it not too obvious: the effect of ambient light feedback on space heating energy consumption
}

\author{
Saskia Maan • Bo Merkus • Jaap Ham • \\ Cees Midden
}

Received: 5 January 2010 / Accepted: 26 August 2010 / Published online: 3 December 2010

(C) The Author(s) 2010. This article is published with open access at Springerlink.com

\begin{abstract}
Earlier research investigating persuasive technology - technology designed to influence human behavior or attitude-indicates that persuasive technology can stimulate energy-efficient behavior. However, most applications of persuasive technology need people's focal attention to be successful, and people may often not have these cognitive resources available. The current research investigates a form of persuasive technology that is less obvious and easier to process: ambient lighting as persuasive technology. In an experimental study, participants could conserve energy while setting temperatures on a central heating panel and receive feedback about their energy consumption in each task. We tested the effect of feedback through a lamp that gradually changed color dependent on energy consumption and compared these effects to more widely used factual feedback. Half of the participants received lighting feedback, and half of the participants received numerical feedback. To investigate whether ambient feedback is easier to process than numerical feedback, half of the participants performed a cognitive load task in addition to the focal task. Results indicated that feedback through lighting has stronger persuasive effects than numerical feedback. Furthermore, ambi-
\end{abstract}

S. Maan · B. Merkus $\cdot$ J. Ham $(\bowtie) \cdot$ C. Midden

Human-Technology Interaction,

Eindhoven University of Technology,

PO Box 513, 5600 MB Eindhoven, The Netherlands

e-mail: j.r.c.ham@tue.nl ent lighting feedback seemed easier to process than numerical feedback because cognitive load interfered with processing numerical feedback, but not with processing lighting feedback. Implications for theory and design of energy consumption feedback systems, persuasive lighting, and (ambient) persuasive technology are discussed.

Keywords Ambient persuasive technology . Feedback · Space heating · Energy consumption · Light

\section{Introduction}

Energy consumption and the emission of greenhouse gasses have been a frequently discussed topic ever since the Kyoto protocol in 1997. The International Energy Outlook 2009 (Energy Information Administration 2009) shows that if no extra measures are taken, energy consumption will rise by $34 \%$ in the next 20 years. The threats of growing $\mathrm{CO}_{2}$ emissions, climate change effects, and the exhaustion of natural resources have urged nations worldwide to seek for substantial reductions in energy consumption. Next to important technological solutions like more efficient systems and devices and the development of renewable energy sources, consumer behavior plays a crucial role in reducing the level of energy consumption.

Influencing consumer behavior to promote energy conservation has become an important target of 
national and international policy efforts. Therefore, the question on which instruments should be applied to promote energy conservation behavior has become highly relevant.

Recent reviews (e.g., Abrahamse et al. 2005; Midden et al. 2007) have evaluated the effects of interventions to promote energy-efficient behavior. In general, mass media public campaigns seem to lack precision in message concreteness and targeting to achieve behavioral change. By contrast, raising people's awareness of energy consumption by providing tailored feedback about their energy consumption (for example in kilowatt-hour) can promote the achievement of behavioral change (see, e.g., Abrahamse et al. 2005; Midden et al. 2007). The results are mixed though. Weak linkages between specific actions and energy outcomes caused by low feedback frequencies (e.g., once per month) and insufficient specificity of the feedback (e.g., household in general vs. specific person or specific devices) are underlying these mixed findings.

Recently, technological solutions have created new opportunities to improve feedback efficacy by embedding feedback in user-system interactions. That is, energy use is in essence always the outcome of an interaction between a user and some energyconsuming device. Intervening in these specific interactions might improve the quality of feedback substantially. Some evidence supports this claim. McCalley and Midden (2002a) demonstrated in several studies that interactive forms of feedback could be effective in enhancing energy-efficient use of devices like washing machines. By adding an energy meter to the user interface of a washing machine, they achieved $18 \%$ of energy conservation both in lab and field studies. Basically, their approach entailed giving numerical feedback in terms of kilowatt-hour consumed as a function of programming choices made by the user, like water temperature, spinning speed, or the duration of the washing cycle. Research by Sauer et al. (2007) also indicates that system-embedded feedback (during operation of a central heating system) can diminish user energy consumption and additionally indicates that users can save energy without lowering comfort levels.

A comparison of studies assessing effects of feedback about energy consumption (Fischer 2008) concludes that it seems clear that feedback stimulates energy savings; Fischer (2008) reports that feedback about energy consumption can lead to energy savings ranging from $1.1 \%$ to over $20 \%$. According to Fischer (2008), good feedback should be based on actual consumption and be detailed and provided frequently. Feedback should be highly interactive and give an appliance specific breakdown. Also, feedback should be presented in an understandable and appealing way.

So, earlier research indicates that feedback about energy consumption can stimulate energy conservation. In other words, giving feedback motivates and stimulates people to conserve energy by changing their energy consumption behavior. Especially feedback that is embedded in user-system interactions can fit these characteristics (be detailed, specific, interactive, appliance specific, etc.). Fogg (2003) refers to this type of technology as persuasive technology which he defines as any "interactive computing system designed to change people's attitudes or behavior."

The main focus of earlier research (e.g., McCalley $\&$ Midden 2002a; Sauer et al. 2007) was on relatively complex feedback that required ample available cognitive capacity to process. However, in many day-to-day situations, people might not be motivated or lack the cognitive capacity to consciously process relatively complex information (see e.g., Bargh 1997). Numerical feedback (e.g., the numbers representing kilowatt-hour consumption) might be that kind of relatively complex information. A form of feedback that is easier to process can be labeled ambient feedback. Ambient displays are designed to be subtle and unobtrusive (Miller \& Rich 2008). Ishii and Ullmer (1997) suggested the use of ambient media such as sound, light, airflow, and water movement to act as background interfaces with cyberspace and work at the periphery of human perception. They also mentioned that even though ambient media are often processed in the background, they can move to the center of attention. Wisnesky and colleagues (1998) supported this definition of ambient technology, by describing ambient technology as being at the "periphery of our attention", with a kind of "subconscious understanding" and possibly involving "all our senses".

In the current research, we will investigate the persuasive effects of a form of ambient feedback that is easier to process. We argue that (interactive) feedback of ambient nature (using lighting) is simpler to process than (interactive) numerical feedback 
because it can be perceived easily without focusing, in contrast to numerical feedback. For example, (part of) the environment of the user can be used for ambient lighting feedback whereas the user needs to focus on digits on a display for numerical feedback.

Earlier research already indicated that energy consumption feedback that does not consists of specific facts but rather of ambient lighting changes can influence consumer behavior (Wisneski et al. 1998; Arroyo et al. 2005; Froehlich 2009). A recent example of this is a device called an energy orb, the color of the orb changed depending on the time-of-use tariff in operation. This type of information helped users save some energy (Martinez 2006), and the usefulness of the device was positively evaluated by users (Martinez 2006; Stein 2004). This study supports the assumption that ambient technologies can reduce the energy consumption of a household.

Furthermore, earlier research indicates that persuasive technology can influence human cognition without receiving any conscious attention at all. That is, Ham et al. (2009) tested the effects of feedback that was presented too shortly to be consciously noticed (subliminal presentation, see e.g., Bargh \& Chartrand 2000) and compared the effects of this feedback to effects of normal feedback or no feedback. Results confirmed that persuasive technology that is not obviously perceived at all can be just as effective as numerical feedback.

To investigate whether lighting feedback is easier to process than numerical feedback, we will employ the insight from earlier research that indicates that the amount of cognitive processing that is needed for a task can be assessed by investigating the effects of adding an additional task to the focal task (Bargh 1997). In psychological literature, such an additional task is called cognitive load. That is, if people need a lot of cognitive processing for a specific focal task, adding an additional task that people have to perform next to the focal task will lead to a decrease in performance (e.g., slower performance) on that focal task. Therefore, we expect that cognitive load will interfere with processing numerical feedback, but not with lighting feedback.

The present research will investigate the influence of ambient feedback versus numerical feedback on energy consumption of space heating of a family home. According to the International Energy Agency (2008), an important role in energy conservation can be identified for households; they account for 30\% of the Dutch energy consumption. Approximately half of this consumption results from space heating.

\section{The current research}

In the current research, the effects of ambient feedback on energy consumption were investigated and compared to the effects of numerical feedback on energy consumption. Ambient feedback was presented in the form of light; colored light was used as feedback to indicate the level of energy consumption. Numerical feedback was presented by the amount of watt consumed. An experiment was set up in which participants had the opportunity to conserve energy in a series of tasks and received feedback about their energy consumption during these tasks. When giving lighting feedback, low consumption was indicated by green lighting and high consumption by red lighting both in various shades. In this way, people could quite easily understand whether a specific lighting (e.g., light green) indicated high or low consumption and less consciously process this information. To keep the two types of feedback comparable, the numerical feedback was presented with two additional numbers indicating low and high energy consumption. This way, the user could determine whether his energy consumption was high or low. The numerical information needs to be processed more consciously because the user himself has to compare his energy consumption with the numbers representing high and low energy consumption.

We expected that feedback through lighting would have stronger persuasive effects (leading to lower energy consumption) than numerical feedback. We also expected that lighting feedback was easier to process than numerical feedback resulting in a decrease in the time used to solve a task for the lighting feedback conditions.

Because we expected lighting feedback to be easier to process than numerical feedback, we also manipulated the cognitive load of the participants. To manipulate cognitive load, half of the participants performed an additional task that consisted of a random number recognition task comparable to successful cognitive load manipulations used by e.g., Marsh and Hicks (1998). This relation between the cognitive load task and the feedback on energy consumption has never been explicitly tested. We 
assume that there is an indirect relation where the feedback represents another stimulus similar to stimuli from the surrounding environment. Because we assume that the ambient feedback will be processed less consciously than the numerical feedback, we expected that for participants processing numerical feedback, performing this additional task would interfere with the persuasive effects of that type of feedback and would result in an increased time to solve the task. At the same time, we expected that for participants processing lighting feedback, performing this additional task would not interfere with the persuasive effects of the feedback and would not result in an increased time to solve the task.

\section{Method}

Participants and design

Participants were mostly university students with the exception of one working participant. Ages of the participants ranged from 18 to 30 years old. In total, 57 participants (39 male and 18 female) participated in a 2 (feedback type: light vs. numerical) $\times 2$ (cognitive load: load vs. no load) between-subject design. Participants were randomly assigned to one of the four conditions.

Materials and procedure

On arrival, participants were welcomed and requested to take a seat in front of the computer followed by the experiment leader starting the virtual central heating interface program and leaving the room. In the experiment, a virtual central heating interface was used to gather data about the participant's energy consumption behavior (see Fig. 1). A model of typical Dutch family housing was programmed to obtain energy consumption data. Energy consumption was calculated taking into account ventilation and transmission losses and characteristics of the different rooms. As input for this model participants had to set the desired temperature for the living room, kitchen, bathroom, toilet, hall, and bed room. The interface was adapted from an existing central heating control unit, the Chronoterm Vision by Honeywell, as can be found in Dutch homes.

After an introduction of the experiment and an explanation of the central heating control unit, participants were requested to pursue two goals. The first goal was to "make sure to set a comfortable room temperature" and the second was to "make sure to use as little energy as possible". The first goal was given to make sure participants would not set extremely low temperatures when pursuing the second goal. Participants were then informed about the workings of the feedback type they were assigned to.

Numerical feedback consisted of the amount of watt representing the energy consumption and a high and low consumption reference value, all presented right from the central heating control unit (see Fig. 1). The reference values differed per scenario. Roughly, the high value represented the amount of watt used when all rooms were heated at $22^{\circ} \mathrm{C}$. The low value represented heating being switched off in every room, or minimal heating for scenarios with low outside temperatures. The same values were used to deter-

Fig. 1 A virtual central heating interface used to gather data about the participant's energy consumption behavior

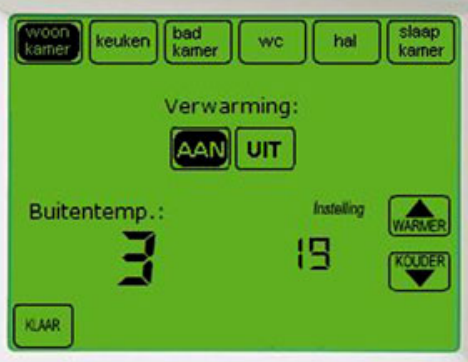

Hoog energieverbruik: 2.328 Watt Laag energieverbruik: 1.011 Watt

Uw energieverbruik is 1.945 Watt 
mine the outer scale of the lighting feedback. Fully red corresponded with the high reference value and fully green with the low values. A high power programmable LED lamp was used to project the light on the wall behind the desk where the participants were seated, see Fig. 2.

After the explanations, participants had to complete two practice trials. In these trials, an outside temperature was presented on the control unit together with a scenario of the following sort: "It is evening and you are at home. The outside temperature is $3^{\circ} \mathrm{C}$ " or "It is afternoon and you are not at home. The outside temperature is $20^{\circ} \mathrm{C}$ ".

Participants in the cognitive load conditions were briefed about the cognitive load task. Participants were asked to press the space bar whenever they heard an odd number through the headphones. Recorded spoken numbers from 1 to 30 were randomly presented with a 3-s interval. Participants could practice for $10 \mathrm{~s}$. The cognitive load task was presented during all ten test trials.

The ten test trials that followed were similar to the practice trials, with scenarios varying outside temperature from $-5^{\circ} \mathrm{C}$ to $19^{\circ} \mathrm{C}$, varying daily periods, and presence or absence of the user.

After the ten test trials, participants were asked to fill out demographic questions. They were then debriefed, paid $€ 5$, and thanked for their participation. The duration of the experiment was no more than $30 \mathrm{~min}$.

Dependent variables were energy consumption and time used to solve a task. The total energy consumption in watts was calculated from the heat losses of the six rooms. The time participants needed to complete a trial was defined as the difference in seconds between the start of the trial and the moment participants hit the "finished" button.

\section{Results}

Averaged energy consumption scores (over the ten tasks) were submitted to a 2 (feedback type: lighting feedback versus numerical feedback) $\times 2$ (cognitive load: load vs. no load) analysis of variance (ANOVA). As expected, participants who had received lighting feedback used a lower amount of energy on average on the tasks (mean $(\mathrm{M})=544 \mathrm{~W}$, standard deviation $(\mathrm{SD})=208)$ than participants who received numerical feedback $(\mathrm{M}=692 \mathrm{~W}, \mathrm{SD}=202), F(1,53)=7.16, p=0.01$ (see Fig. 3). This effect indicates a reduction of $21 \%$ in energy consumption for participants who received lighting feedback as compared to participants who received numerical feedback. This analysis did not indicate the expected interaction of feedback type $\times$ cognitive load, $F<1$ nor a main effect of cognitive load, $F<1$.

To analyze the other dependent variable - time to solve a task: an average of the time a participant needed to program the control unit on each of the ten tasks - we submitted time to solve a task to the same $2 \times 2$ ANOVA. As is often the case when analyzing response times (Ratcliff 1993), a few participants scored outliers (more than two SDs from the average response time) on this measure. Therefore, as advised

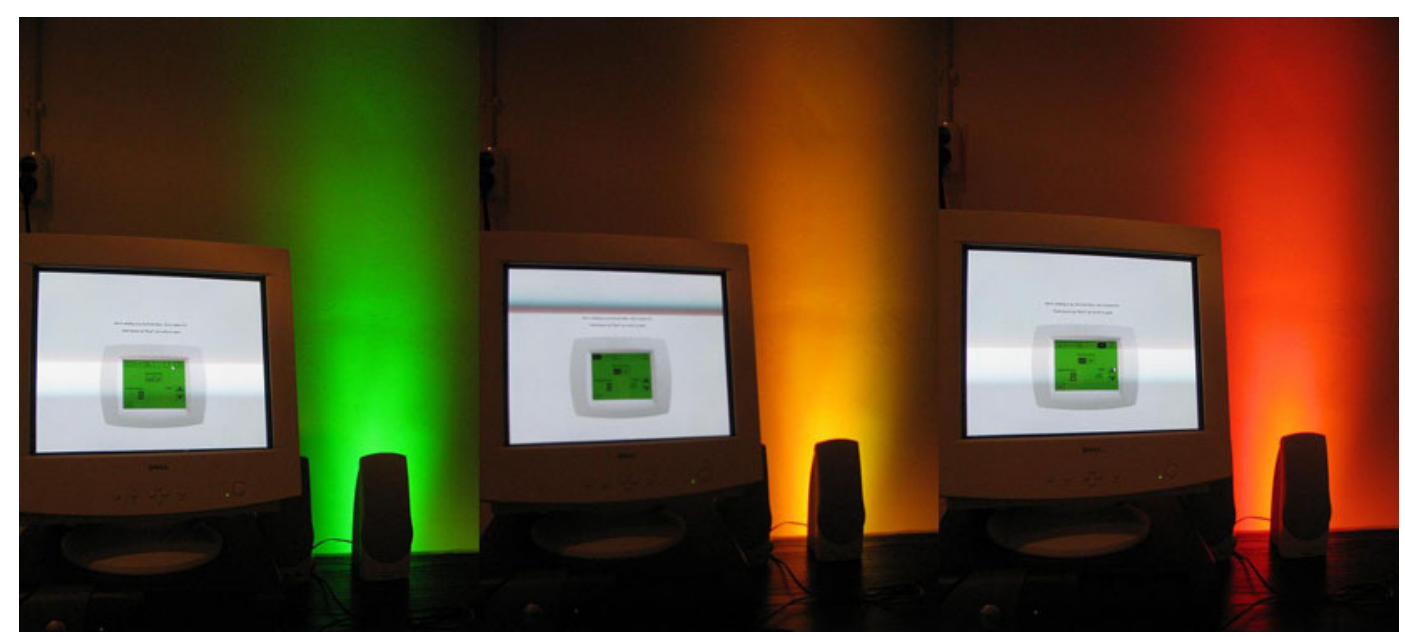

Fig. 2 A high power programmable LED lamp projecting light on the wall behind the participant's desk indicating low (left picture), medium (middle picture), or high (right picture) energy consumption 


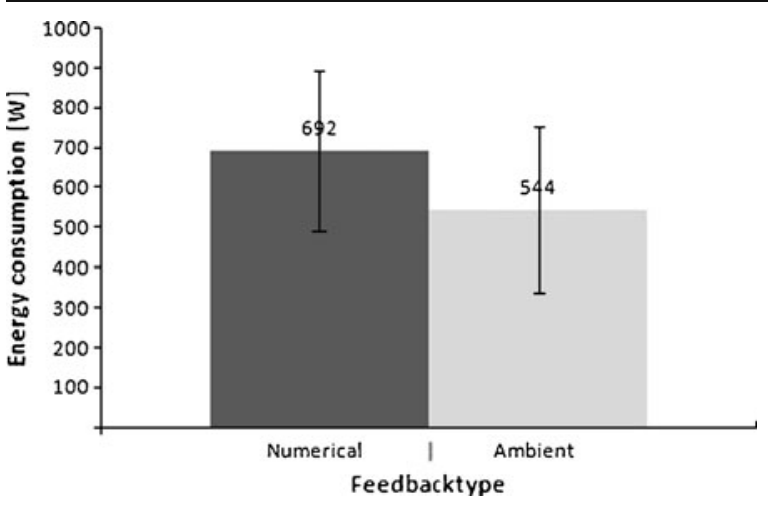

Fig. 3 Averaged energy consumption by feedback type

by Ratcliff (1993), we removed these (four out of 57) participants from this analysis. As expected, results indicated a main effect of feedback type on time to solve a task, $F(1,49)=11.76, p=0.001$. That is, participants who received numerical feedback needed more time to solve a task $(\mathrm{M}=45 \mathrm{~s}$, standard error $(\mathrm{SE})=12)$ than participants who received lighting feedback $(M=36 \mathrm{~s}, \mathrm{SE}=9)$. Also, results indicated that participants who performed the additional cognitive load task needed more time to solve a task $(\mathrm{M}=45, \mathrm{SD}=14)$ than participants who did not perform this additional load task $(\mathrm{M}=37, \mathrm{SD}=7)$, indicated by a main effect of cognitive load on time to solve a task, $F(1,49)=7.77, p=0.008$. Most importantly, results also indicated the expected interaction of feedback type $\times$ cognitive load, $F(1,49)=4.50$, $p=0.039$ (see Fig. 4). That is, participants who received numerical feedback needed more time to program the thermostat under cognitive load $(\mathrm{M}=52.0 \mathrm{~s}, \mathrm{SD}=13.5)$ than without cognitive load $(\mathrm{M}=38.7 \mathrm{~s}, \mathrm{SD}=7.0), \quad F(1,50)=9.63, p=0.003$ whereas this difference was not found for participants who received lighting feedback $(\mathrm{M}=36.9, \mathrm{SD}=10.4$, for participants under cognitive load versus $M=35.1$, $\mathrm{SD}=7.5$, for participants without cognitive load), $F<1$. Thereby, these results indicate that lighting feedback was easier to process. Furthermore, they suggest that participants compensated for the cognitive load by taking more time to finish the trials.

\section{Discussion}

The aim of this study was to test the persuasive effects of lighting feedback as ambient persuasive technology in stimulating energy consumption behavior and compare them to the persuasive effects of a form of persuasive technology that needs more focal attention, that is, numerical feedback. Confirming expectations, lighting feedback led to $21 \%$ lower energy consumption than numerical feedback. Furthermore, lighting feedback was expected to be easier to process than numerical feedback. This expectation was confirmed: participants who received lighting feedback finished trials quicker than participants who received numerical feedback. Furthermore, in strong corroboration of our hypothesis, current results suggest that for participants processing numerical feedback, performing an additional task led to slower processing of that feedback. For participants processing lighting feedback, results suggest that adding cognitive load did not lead to slower processing. This finding confirms our suggestion that lighting feedback is easier to process and use than numerical feedback.

The current results did not indicate that for participants processing numerical feedback, performing an additional task led to a lower energy consumption than when not doing an additional task. An important reason for this might be that the setup of the current study may not have been ideal for finding such an effect because of the lack of time constraints when setting the control unit. That is, without time constraints, participants who received numerical feedback and who performed an additional task may have been able to use more time to set the control unit (and did so, as indicated by the analysis of time to solve a task). It seems quite straightforward that these participants used this additional time to process the numerical

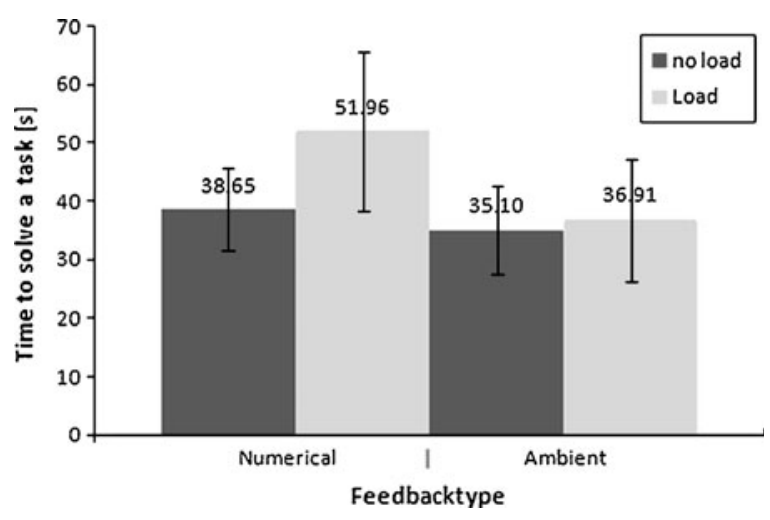

Fig. 4 Time to solve a task by feedback type and cognitive load 
feedback. Thereby, these participants may have processed the numerical feedback well, even though they also had to spend processing capacity on the additional cognitive load task. Future research might continue the investigation of whether cognitive load can increase energy consumption for numerical feedback. Importantly, the current results indicate that setting time constraints might be important to find those effects.

Future research might also investigate the effects of ambient feedback in a field setting. Interestingly, research by McCalley and Midden (2002a) indicated similar energy saving results after interactive feedback in a field study as in a lab study (McCalley \& Midden 2002b). This suggests that effects of ambient feedback might also be comparable in field and lab settings. Also, future research might investigate issues related to somatic experiences. That is, it might be very interesting to compare effects of ambient and also numerical feedback to effects of actually experiencing the feeling of warmth and heating caused by settings of a heating system. Finally, future research might compare the effects of ambient feedback to the effects of focal feedback. The goal of the current research was to compare ambient to numerical feedback, and future research might compare the ease of processing of ambient feedback to the ease of processing of focal feedback.

The current study focuses on the possible effect of ambient feedback on energy consumption. However, besides the expected positive effect on energy consumption, there are privacy and ethical implications that need to be considered when dealing with ambient technologies (Bohn et al. 2005). The application discussed in this article implies a constant monitoring of energy consumption data. Application of systems like these in future households requires careful consideration of privacy issues like to whom the data are accessible, how the data are stored, and for how long they are stored. Furthermore, ambient persuasive technology is designed to change people attitudes or behavior in an unobtrusive way. Within the scope of this article, an ethical discussion on this topic carries too far. However, in the design and application of ambient persuasive technologies, one should at all times be aware of issues concerning the right of self-determination and personal autonomy of the user. In addition, there might be adverse effects of providing feedback, in more focal form or in more ambient form. For example, in general, persuasive technology might give rise to reactance (Brehm 1966, see also, Roubroeks et al. 2009). Also, lighting feedback could help people conserve energy and thereby save money, adding persuasive technology to household appliances may increase financial costs, making economic analyses needed to assess overall effects.

The present research shows that lighting feedback is more effective than numerical feedback. Besides easier processing, this may be caused by an implicit evaluation of the energy consumption which took place in the lighting feedback. When lighting feedback was given, the color of the light indicated good or bad energy consumption behavior. In contrast, when numerical feedback was given, participants had to do this evaluation by themselves by comparing their energy consumption with the two reference values. In future work, the effects of easier processing and implicit evaluation could be disentangled.

The main effect of feedback type on energy consumption showed that participants understood the meaning of the colors used for the lighting feedback. However, the used colors might not be optimal with regard to their effect on energy consumption.

Although the meaning of green and red is supposed to be generally understood, future research should explore whether these colors are most effective for giving feedback on energy consumption. A starting point for this research could be environmental psychology literature on the effect of light on humans. For example, recent research (Mehta \& Zhu 2009) suggested that red (versus blue) induces avoidance (versus approach) motivation, and that red enhances performance on a detail-oriented task. Also, Kwallek (Kwallek et al. 1988) showed that people in a red surrounding scored higher in stress and anxiety measures, and other articles find a strong effect of light on arousal (e.g., Baron et al. 1992; Bellizzi \& Hite 1992; Kallman \& Isaac 1977). In addition, future research might investigate whether colors are effective only for giving energy consumption feedback or whether they could also be employed for giving other kinds of feedback.

In the introduction, we claim that lighting feedback is a form of ambient technology. This could be argued given the rather obvious projection of the light against the wall behind the desk. Since ambient technologies are supposed to be processed subconsciously and/or in the periphery of attention (Wisneski et al. 1998), 
one would expect ambient technologies to be easier to process. The present study provides support for that claim.

Overall, the current research indicates that diffuse lighting can be used successfully as persuasive technology. These technologies can be incorporated into everyday life in many forms to change different types of behavior or attitudes. For example, the data about energy consumption provided by smart meters could be used to deliver interactive lighting feedback in the living room. The current research suggests that such an application could successfully influence energy consumption behavior even when users do not spend cognitive attention to this lighting feedback. The feedback given in the current research changes color rapidly whereas in field situations, changes in lighting might be of a different nature (e.g., more subtle), which future research could investigate. This could also determine the optimal positioning of the lighting feedback. The current research indicates that lighting can have a particular aptitude as a medium for persuasive communications. The current research suggests that persuasive lighting can have stronger persuasive effects than other forms of persuasive technologies (i.e., numerical and factual), especially under (day-to-day) circumstances of high cognitive load.

In addition, we argue that lighting has specific qualities that make it particularly suitable for providing user feedback. Lighting can be very cheap, is easy to install, lighting can be very energy-friendly, lighting can be seen by other people present in a room as well (inducing social pressure as a persuasive mechanism), and lighting might have an emotional appeal or even direct emotional effects. Also, the low conspicuity of light and color changes set lighting apart from other feedback media.

This research showed that ambient lighting feedback has a positive effect on energy efficiency and is easier to process. In addition, this study shows the relevance of the use of light in ambient technologies and the importance of the use of ambient feedback to give feedback on energy consumption. This could have implications for the design of future household appliances and space heating systems because the addition of ambient feedback into these devices has great potential to reduce household energy consumption and greenhouse gasses.
Acknowledgement We would like to thank Martin Boschman for his technical assistance.

Open Access This article is distributed under the terms of the Creative Commons Attribution Noncommercial License which permits any noncommercial use, distribution, and reproduction in any medium, provided the original author(s) and source are credited.

\section{References}

Abrahamse, W., Steg, L., Vlek, C., \& Rothengatter, T. (2005). A review of intervention studies aimed at household energy conservation. Journal of Environmental Psychology, 25, 273-291.

Arroyo, E., Bonanni, L., Selker, T. (2005). Waterbot: exploring feedback and persuasive techniques at the sink. Proceedings of the SIGCHI conference on human factors in computing systems (CHI 2005), 631-639.

Bargh, J. A. (1997). The automaticity of everyday life. In R. S. Wyer Jr. (Ed.), The automaticity of everyday life: advances in social cognition (Vol. 10, pp. 1-61). Mahwah, NJ: Erlbaum.

Bargh, J. A., \& Chartrand, T. L. (2000). The mind in the middle: a practical guide to priming and automaticity research. In H. Reis \& C. Judd (Eds.), Handbook of research methods in social and personality psychology (pp. 253-285). New York: Cambridge University Press.

Baron, R. A., Rea, M. S., \& Daniels, S. G. (1992). Effects of indoor lightning (illuminance and spectral distribution) on the performance of cognitive tasks and interpersonal behaviors: the potential mediating role of positive affect. Motivation and Emotion, 16, 1-33.

Bellizzi, J. A., \& Hite, R. E. (1992). Environmental color, consumer feelings, and purchase likelihood. Psychology \& Marketing, 9, 347-363.

Bohn, J., Coroama, V., Langheinrich, M., Mattern, F., \& Rohs, M. (2005). Social, economic, and ethical implications of ambient intelligence and ubiquitous computing. In: W. Weber, J. Rabaey, E. Aarts (Eds.), Ambient Intelligence (pp. 5-29). Springer-Verlag.

Brehm, J. W. (1966). A theory of psychological reactance. New York: Academic.

Energy Information Administration (2009). International Energy Outlook 2009.

Fischer, C. (2008). Feedback on household electricity consumption: a tool for saving energy? Energy Efficiency, 1, 79-104.

Fogg, B. J. (2003). Persuasive technology: using computers to change what we think and do. San Francisco: Morgan Kaufmann.

Froehlich, J. (2009). Promoting energy efficient behaviors in the home through feedback: the role of human-computer interaction. HCIC 2009 Workshop released as a UW tech note \#09-02-01.

Ham, J., Midden, C., \& Beute, F. (2009). Unconscious persuasion by ambient persuasive technology: evidence for the effectiveness of subliminal feedback. Conference proceedings of Artificial Intelligence and Simulation of Behaviour, 2009, Edinburgh, UK. 
Hemphill, M. (1996). A note on adults' color-emotion associations. The Journal of Genetic Psychology, 157, 275-280.

International Energy Agency (IEA). (2008). Worldwide trends in energy use and efficiency. Paris: IEA Publications.

Ishii H., Ullmer, B. (1997). Tangible bits: towards seamless interfaces between people, bits and atoms. Proceedings of CHI' 97 (pp 234-241), ACM Press.

Kallman, W. M., \& Isaac, W. (1977). Altering arousal in humans by varying ambient sensory condition. Perceptual and Motor Skills, 44, 48-64.

Kwallek, N., Lewis, C. M., \& Robbins, A. S. (1988). Effects of office interior color on workers' mood and productivity. Perceptual and Motor Skills, 66, 123-128.

Marsh, R. L., \& Hicks, J. L. (1998). Event-based prospective memory and executive control of working memory. Journal of Experimental Psychology. Learning, Memory, and Cognition, 24, 336-349.

Martinez, M.S. (2006). "Residential demand response technologies: A consumer's guide." Presentation at National Town Meeting and Symposium on Demand Response.

McCalley, L. T., \& Midden, C. J. H. (2002a). Energy conservation through product integrated feedback: the roles of goal-setting and social orientation. Journal of Economic Psychology, 23, 589-603.

McCalley, L. T., \& Midden, C. J. H. (2002b). Getting energy conservation feedback to work: a new understanding. In Bartels \& Nelissen (Eds.), Marketing for sustainability.
Towards transactional policy-making (pp. 383-395). Amsterdam: IOS Press.

Mehta, R., \& Zhu, R. J. (2009). Blue or red? Exploring the effect of color on cognitive task performances. Science, 323, 1226-1229.

Midden, C. J. H., Kaiser, F. G., \& McCalley, L. T. (2007). Technology's four roles in understanding individuals' conservation of natural resources. Journal of Social Issues, 63, 155.

Miller, T., \& Rich, P. (2008). ADAPT - audience design of ambient persuasive technology. Mentored Advanced Project (MAP) Report, August 2008.

Ratcliff, R. (1993). Methods for dealing with reaction time outliers. Psychological Bulletin, 114, 510-532.

Roubroeks, M., Midden, C., \& Ham, J. (2009). Does it make a difference who says it? Exploring the role of a social agent for psychological reactance. Conference proceedings of Persuasive 2009, Claremont, USA.

Sauer, J., Schmeink, C., \& Wastell, D. (2007). Feedback quality and environmentally friendly use of domestic central heating systems. Ergonomics, 50, 795-813.

Stein, L.F. (2004). California information display pilot: Technology assessment. Final Report by Primen to Southern California Edison.

Wisneski, C., Ishii, H., Dahley, A., Gorbet, M.G., Brave, S., Ullmer, B., \& Yarin, P. (1998). Ambient displays. In N.A. Streitz, S. Konomi, H.-J. Burkhardt, (Eds.) CoBuild 1998. LNCS, 1370. Springer. 